\title{
Surgical Services in Devolved Manchester
}

\author{
Chelliah R Selvasekar MD, FRCSEd (Gen), MFSTEd, Pg Cert (Med Ed), MBA \\ Consultant General, Colorectal, Laparoscopic \& Robotic Surgeon, The Christie NHS \\ Foundation Trust, Manchester. M20 4BX; MCh Pathway lead for Surgical specialities, Hon Sr. \\ Lecturer, Edge Hill University \\ nselvasekar@gmail.com
}

"There is a culture change required at the grass root level for this project to succeed and clinical engagement is paramount."

Cite as: Selvasekar, CR. (2017) Surgical Services in Devolved Manchester. Sushruta 10(1): 11 DOI: $10.38192 / 10.1 .4$

Devolution Manchester is a pilot project to provide devolved health and social care to the population of Greater Manchester under the leadership of the Mayor. Department of Health has provided $f 6$ billion towards this project to provide health and social care for a population of 2.8 million. This project aims at integrating health and social care. Currently there is fragmentation and the ultimate aim of this project is to bring a system wide change and to have health and social care under one roof. It also aims at decentralization of health care where decisions are made locally without interference from the central government. This ultimately is aimed at providing safe and high quality care, if implemented well.

\section{Surgical Services:}

At present specialist general surgical management is provided in most of the hospitals in Greater Manchester. With the unified approach, acute abdominal surgery is planned to be offered at high quality in four major sites. These four primary sites include The Royal Oldham Hospital, The Salford Royal NHS Foundation Trust, Manchester University NHS Foundation Trust and Stepping hill Hospital. These four sites are also the sites where major emergency surgery will be offered under the healthier together initiative. The other sites currently offering surgery will be downgraded to provide basic elective care with support from these four major sites. To be successful this programme needs investment, patient focused standardized pathways and a major culture change among the clinicians to work at multiple sites with flexible job plans.

\section{Specialist surgical services:}

Devolution Manchester project allows for centralization or regionalization of specialized services. There is high quality evidence in complex gastrointestinal surgery such as oesophagogastric surgery, pancreatic and advanced pelvic surgery where high volume and improved outcome relationship has been demonstrated. Devolution Manchester with its 
unified approach allows better infrastructure to be placed in a centralized unit with clinicians dealing with complex issues on a regular basis to provide improved outcomes for the local population.

\section{Research:}

This unified project allows recruitment of patients for clinical research easier with various organizations collaborating such as the Manchester academic health science centre (MAHSC) which includes partnerships with Manchester cancer research centre (MCRC), major provider trusts and commissioning groups in Manchester. This provides an opportunity to translate newer cutting edge treatments to be brought to forefront clinical use sooner than what is currently possible and hopefully eliminate the various bureaucratic processes.

\section{Education and Training:}

There needs to be a radical review of the current models of surgical training whereby the trainees are attached to one hospital and have varying levels of service and educational commitments. To train surgeons for the future, we need to allow trainees to have flexible contracts with hospitals in the Greater Manchester and training based on the trainee needs on a competitive basis. We also need to develop a robust mentoring and support programme to provide optimal guidance based on the educational needs which can be addressed in a structured and systematic process based on the performance.

\section{Challenges:}

NHS has gone through various reorganizations and this project signals reorganization without properly evaluating the effects of the previous restructuring. Unfortunately this seems to be recurring theme of the modern NHS! Various well thought initiatives are discussed but not implemented and there is virtually no learning from the failures. There is a culture change required at the grass root level for this project to succeed and clinical engagement is paramount. Unfortunately there has not been public consultation nor has there been effective communications of the various actions taken so far. Finally for this system wide change to be successfully implemented $£ 6$ billion is not enough!

\section{Conclusion:}

Devolution Manchester is a radical system wide change in providing integrated care allowing improved access for patients. It aims to eliminate variation, facilitate standardization and equitable care. It is possible for patients to have care closer to home for common conditions and to have specialized care centrally at high volume well-resourced centres. This project allows for the use of modern information technology and by working together various organizations can engage in developing patient specific pathways to provide high quality equitable care for the local population based on the local needs. This project has the potential to provide sustainable health and social care to Greater Manchester but needs a thoughtful implementation.

https://www.gmhsc.org.uk/ 